\title{
ON THE FIRST COUNTABILITY OF HYPERSPACES
}

\author{
TAKEMI MIZOKAMI
}

ABSTRACT. In this paper, the heredity of first countability of a space $X$ on the hyperspace $\mathcal{C}(X)$ of nonempty compact subsets of $X$ with the finite topology is investigated and some variations are given.

Introduction and terminology. In [4] Smithson gives an example which shows that the hyperspace $C(X)$ of nonempty compact subsets of a first countable space $X$ need not be first countable with respect to the finite topology in the sense of [2], and he proves that a necessary and sufficient condition for the first countability of $\mathcal{C}(X)$ is that $X$ is compactly second countable. The author, in Theorem 3, states the characterization of such spaces, answering question 3 in [4]. In Theorem 1, the characterization of open images of locally compact metric spaces is given. Theorems 2, 4 and 5 give some variations to such spaces. All spaces in the sequel are assumed to be Hausdorff, and all mappings are continuous. $N$ always denotes the set of positive integers.

Before stating results, we give some notation and terminology used here. We define the properties of a space $X$ by (M), (C), (S), (SC) and (G) as follows: (M): Every compact subspace of $X$ is metrizable. (C): Every compact subset of $X$ is of countable character in the sense of [5], i.e., every compact subset $K$ of $X$ has an open collection $\left\{U_{n}: n \in N\right\}$ with $K \subset U_{n}$ for every $n$ such that if $K \subset G$ with $G$ open, then $U_{n} \subset G$ for some $n$. (S): Every compact subset of $X$ is separable. (SC): Every compact subspace of $X$ is second countable. (G): Every compact subset $K$ of $X$ has a countable open collection $\mathcal{P}(K)$ covering $K$ such that if $p, q \in K$ are distinct, then $p \in P$, $q \notin P$ for some $P \in \mathscr{P}(K)$. Note that the term compactly first countable in [4] is nothing but (C). According to Michael and Nagami [2], a compact subset $K$ of a space $X$ has a countable $X$-base for $K$ if there exists a countable open collection $\mathscr{B}(K)$ such that whenever $p \in G \cap K$ with $G$ open, $p \in B \subset G$ for some $B \in \mathscr{B}(K)$. This is equivalent with the term compactly second countable in [4]. A mapping $f: X \rightarrow Y$ is said to be compact-covering if for every compact set $K$ of $Y$ there exists a compact set $L$ of $X$ such that $f(L)=K$.

Received by the editors May 9, 1977 and, in revised form, June 17, 1977.

AMS (MOS) subject classifications (1970). Primary 54B20.

Key words and phrases. Hyperspaces, the finite topology, countable character, open and compact-covering mappings.

() American Mathematical Society 1977 
LEMMA 1. Suppose $f$ is an open mapping from a space $T$ onto a space $X$. If $T$ is a locally compact metrizable space, then the mapping $f^{*}: \mathcal{C}(T) \rightarrow \mathcal{C}(X)$, defined by $f^{*}(K)=f(K)$ for every $K \in \mathcal{C}(T)$, [2] is onto and open.

Proof. Firstly, we shall prove the following: If $K \subset f(U)$ with $K$ compact in $X$ and $U$ open in $T$, then there exists a compact set $L \subset U$ with $f(L)=K$. This implies that $f$ is compact-covering. To see this, take a point $p(y) \in U \cap$ $f^{-1}(y)$ for each $y \in K$. Since $T$ is locally compact and regular, there exists a compact nbd (= neighborhood) $V(p(Y))$ of $p(y)$ such that $V(p(y)) \subset U . f$ open implies that $f(V(p(y)))$ is a nbd of $y$ in $X$. Since $K$ is compact, there exists a finite subset $K_{0}$ of $K$ such that

$$
K \subset \cup\left\{f(V(p(y))): y \in K_{0}\right\} \subset f(U) .
$$

Put $V=\bigcup\left\{V(p(y)): y \in K_{0}\right\}$. Then $V$ is compact. Put $L=f^{-1}(K) \cap V$. Then $L$ is a compact set with the desired property. Now we shall show that $f^{*}$ is open. To see this, it suffices to prove that $f^{*}\left(\left\langle U_{1}, \ldots, U_{n}\right\rangle\right)$ is open in $\mathcal{C}(X)$ for all open sets $U_{1}, \ldots, U_{n}$ of $T$. Let $K \in\left\langle U_{1}, \ldots, U_{n}\right\rangle$; then we show that $f(K) \in \operatorname{Int}\left(f^{*}\left(\left\langle U_{1}, \ldots, U_{n}\right\rangle\right)\right) . K \in\left\langle U_{1}, \ldots, U_{n}\right\rangle$ means $f(K) \in$ $\left\langle f\left(U_{1}\right), \ldots, f\left(U_{n}\right)\right\rangle$ open in $\mathcal{C}(X)$. Let $L$ be any element of $\left\langle f\left(U_{1}\right), \ldots, f\left(U_{n}\right)\right\rangle$, that is, $L \subset f\left(\cup U_{j}\right)$ and $L \cap f\left(U_{j}\right) \neq \varnothing$ for each $j$. By that above statement, there exists a compact set $M \subset \cup U_{j}$ with $f(M)=L$. Take $x_{j} \in f^{-1}(L) \cap U_{j}$, and put $M^{\prime}=M \cup\left\{x_{j}: j=1, \ldots, n\right\}$. Then $M^{\prime}$ is compact and $M^{\prime} \in\left\langle U_{1}, \ldots, U_{n}\right\rangle$, and $f\left(M^{\prime}\right)=L$, proving that $f^{*}$ is open. Since $f$ is compact-covering, $f^{*}$ is onto. By $(5.10 .1)$ in [1], $f^{*}$ is continuous. Thus we complete the proof.

COROLlaRY. Any open mapping from a locally compact regular space is compact-covering.

Lemma 2 (Ponomarev [3]). A space $S$ is first countable iff $S$ is an open image of a metric space.

THEOREM 1. For a space $X$ the following are equivalent:

(1.1) $X$ is a first countable, locally compact regular space with (M).

(1.2) There exists an open compact-covering mapping $f$ from a locally compact metrizable space $T$ onto $X$.

(1.3) There exists an open mapping $f$ from a locally compact metrizable space $T$ onto $X$.

(1.4) $\mathcal{C}(X)$ is an open image of a locally compact space.

(1.5) $\mathcal{C}(X)$ is a first countable, locally compact regular space with (M).

Proof. (1.1) $\rightarrow(1.2)$ : Suppose $X$ is a first countable regular space with (M). Let $K$ be any nonempty compact subset of $X$. Then there exists an open set $V(K)$ containing $K$ such that $\overline{V(K)}$ is compact. Let $\left\{V\left(K_{\alpha}\right)\right\}$ be the totality of such open sets for all compact subsets of $X$. Let $T$ be the topological disjoint sum of all $V\left(K_{\alpha}\right)$ with the relative topology, and define a mapping $f$ from $T$ onto $X$ by a natural way. Obviously $f$ is compact-covering and 
continuous. Since $X$ is regular, $T$ is locally compact and metrizable. To see that $f$ is open, let $G$ be any open set in $T, p \in G$ and $f(p)=y$. Then there exists $V\left(K_{\alpha}\right)$ with $p \in V\left(K_{\alpha}\right)$. Let $\left\{V_{n}(p)\right\}$ be a local base of $p$ in $X$. Since $V\left(K_{\alpha}\right)$ is open in $X$, there exists $V_{n}(p)$ such that $V_{n}(p) \subset G \cap V(K)$, implying $V_{n}(p) \subset f(G)$. Hence $f(G)$ is open, completing the proof of $(1.1) \rightarrow$ (1.2). (1.2) $\rightarrow$ (1.3) is trivial. (1.3) $\rightarrow$ (1.4): Suppose we are given $f: T \rightarrow X$ satisfying the conditions stated in (1.3). Define a mapping $f^{*}: \mathcal{C}(T) \rightarrow \mathcal{C}(X)$ by $f^{*}(K)=f(K)$ for every $K \in \mathcal{C}(T)$. Note that $\mathcal{C}(T)$ is a locally compact metrizable space by (4.9.12) and (4.9.13) in [1]. By Lemma $1, f^{*}$ is open and onto. This implies the truth of $(1.3) \rightarrow(1.4)$. (1.4) $\rightarrow(1.5)$ is due to Lemma 2 and the Corollary to Lemma $1 .(1.5) \rightarrow(1.1)$ : By (4.9.12) and (4.9.13) $X$ is a first countable, locally compact regular space. Let $K$ be any nonempty compact set of $X$. Then $\hat{K}=\{\{x\}: x \in K\}$ is compact in $\mathcal{C}(X)$, and is homeomorphic to $K$. Since $\hat{K}$ is metrizable, so is $K$. Thus $X$ has (M).

This theorem gives a characterization of spaces that are open images of locally compact metric spaces. The content is forseeable in the light of the later results of this paper, but the proof is somewhat different.

THEOREM 2. For a space $X$ the following are equivalent:

(2.1) $X$ is a first countable space with (M) and (C).

(2.2) $X$ is a space with $(\mathrm{M})$ and $(\mathrm{C})$.

(2.3) $X$ is a space with (C) and (SC).

(2.4) $X$ is a space with $(\mathrm{C})$ and $(\mathrm{G})$.

(2.5) $X$ is an open compact-covering image of a metric space.

(2.6) Every compact subset of $X$ has a countable $X$-base.

Proof. (2.1) $\rightleftarrows(2.2) \rightleftarrows(2.3)$ are trivial. $(2.2) \rightleftarrows(2.5)$ is Theorem 1.2 in [2], and $(2.2) \rightleftarrows(2.6)$ is Lemma 3.1 in [2]. (2.4) $\rightarrow(2.2)$ is obvious if we note that every compact subspace with $(\mathrm{G})$ is metrizable. $(2.6) \rightarrow(2.4)$ is trivial.

THEOREM 3. For a space $X$ the following are equivalent:

(3.1) $\mathcal{C}(X)$ is first countable.

(3.2) $X$ is a space with $(\mathrm{C})$ and $(\mathrm{S})$.

Proof. (3.1) $\rightarrow$ (3.2): It follows from Theorem 2 in [4] that $X$ has (S). Therefore it remains to prove that $X$ has (C). Let $K$ be any nonempty compact set of $X$. Let $\left\{\mathscr{U}_{n}: n \in N\right\}$ be a local base of $K$ in $\mathcal{C}(X)$; without loss of generality we can denote each $\mathcal{Q}_{n}$ by $\left\langle U_{n 1}, \ldots, U_{n k_{n}}\right\rangle$ where each $U_{n j}$ is open in $X$. Put $V_{n}=\cup\left\{U_{n j}: j=1, \ldots, k_{n}\right\}$. Then $V_{n}$ is an open set containing $K$. Let $G$ be any open set containing $K$. Then $K \subset\langle G\rangle$ in $\mathcal{C}(X)$. Therefore $\mathscr{Q}_{n} \subset\langle G\rangle$ for some $n$. It is obvious that $V_{n} \subset G$. Thus $K$ is of countable character. (3.2) $\rightarrow(3.1)$ : Let $K \in \mathcal{C}(X)$. Then by (S) there exists a countable dense subset $\left\{x_{m}: m \in \mathfrak{T}\right\}$ of $K$. Since $X$ is first countable, there exists for each $m$ a local base $\left\{V_{n}\left(x_{m}\right): n \in N\right\}$ of $x_{m}$ in $X . K$ is of countable character, and therefore there exists a countable open nbd base $\left\{U_{i}: i \in N\right\}$ of $K$ in $X$. Put $\mathscr{V}=\left\{V_{n}\left(x_{m}\right): n, m \in N\right\}$. Let $\left\{\mathscr{V}_{i}: i \in N\right\}$ be the totality of 
finite subcollections of $\mathscr{V}$; then put for each $i, j \in N, \mathscr{V}_{i}\left(U_{j}\right)=\{K \in$ $\bigodot(X): K \subset U_{j}$ and $K \cap V \neq \varnothing$ for every $\left.V \in \mathcal{V}_{i}\right\}$. Then $\mathcal{V}_{i}\left(U_{j}\right)$ is an open set in $\mathcal{C}(X)$ containing $K$. We shall show that $\left\{\mathscr{V}_{i}\left(U_{j}\right): i, j \in N\right\}$ is a local base of $K$ in $\mathcal{C}(X)$. Suppose $K \in\left\langle W_{1}, \ldots, W_{m}\right\rangle$, where each $W_{i}$ is open in X. $K \subset \cup W_{i}$ implies that $K \subset U_{j} \subset \cup W_{i}$ for some $j$. Since $K \cap W_{i} \neq \varnothing$, there exists a point $x_{m(i)}$ and an integer $k(i)$ such that $V_{k(i)}\left(x_{m(i)}\right) \subset W_{i}$. Then $\left\{V_{k(i)}: i=1, \ldots, m\right\}=\mathcal{V}_{t}$ for some $t \in N$. It is easily seen that $K \in \mathcal{V}_{t}\left(U_{j}\right)$ $\subset\left\langle W_{1}, \ldots, W_{m}\right\rangle$.

Theorem 3 answers question 3 proposed by Sinithson in [4].

In the next lemma, a space $X$ is said to have a $G_{\delta}$-diagonal if the diagonal set of $X \times X$ is a $G_{\delta}$-set, and this is equivalent with the existence of a sequence $\left\{\mathscr{U}_{n}: n \in N\right\}$ of open covers of $X$ with $\cap S\left(p, \mathscr{U}_{n}\right)=\{p\}$ for every point $p \in X$. If a space $X$ has a sequence $\left\{\mathcal{Q}_{n}: n \in N\right\}$ of open covers of $X$ with $\cap S\left(p, \mathscr{U}_{n}\right)=\{p\}$ for every point $p \in X$, then $X$ is said to have a $\overline{G_{\delta}}$-diagonal.

LEMMA 3. If $X$ has a $\overline{G_{\delta}}$-diagonal, then $C(X)$ has a $G_{\delta}$-diagonal.

Proof. Let $\left\{\mathscr{Q}_{n}: n \in N\right\}$ be a sequence of open covers of $X$ such that $\cap \overline{S\left(p, \mathscr{Q}_{n}\right)}=\{p\}$ for every point $p \in X$. Construct $\left\langle\mathscr{Q}_{n}\right\rangle=$ $\left\{\left\langle U_{1}, \ldots, U_{m}\right\rangle: U_{1}, \ldots, U_{m} \in \mathscr{Q}_{n}, m=1,2, \ldots\right\}$. Then $\left\langle\mathscr{U}_{n}\right\rangle$ is an open cover of $\mathcal{C}(X)$. Let $K_{1}, K_{2}$ be distinct points in $\mathcal{C}(X)$. Then there exists a point $p \in K_{1}-K_{2}$ without loss of generality. This means $S\left(p, \mathscr{U}_{n}\right) \cap K_{2}=$ $\varnothing$ for some $n$. For this $n$, suppose $K_{1}, K_{2} \in\left\langle U_{1}, \ldots, U_{m}\right\rangle$ for $U_{1}, \ldots, U_{m} \in$ $\mathcal{Q}_{n}$. $p \in K_{1}$ implies $p \in U_{j}$ for some $j$. On the other hand, $K_{2} \in\left\langle U_{1}, \ldots, U_{m}\right\rangle$ implies $K_{2} \cap U_{j} \neq \varnothing$. This is a contradiction to $S\left(p, \mathcal{U}_{n}\right) \cap K_{2}=\varnothing$. Hence we have $K_{1} \notin S\left(K_{2},\left\langle\mathcal{U}_{n}\right\rangle\right)$.

THEOREM 4. If a space $X$ has a $\overline{G_{\delta}}$-diagonal and property $(\mathrm{C})$, then $\mathrm{C}(X)$ is a first countable space with a $G_{\delta}$-diagonal.

Proof. Note that every compact space with a $G_{\delta}$-diagonal is metrizable, and necessarily separable. This combined with Theorem 3 and Lemma 3 implies Theorem 4.

The following theorem is a variation to the case of point-countable base. The proof is analogous to the preceding case, or routine, and is omitted.

THEOREM 5. For a space $X$ the following are equivalent:

(5.1) $\mathcal{C}(X)$ has a point-countable base.

(5.2) $X$ has a point-countable base.

(5.3) There exists an open collection $\mathscr{P}$ such that for each compact set $K$ of $X$, $\mathcal{P}(K)=\{P \in \mathcal{P}: K \cap P \neq \varnothing\}$ is a countable $X$-base for $K$ in $X$.

(5.4) There exists an open s-mapping from a metric space onto $X$.

For the definition of an $s$-mapping and the equivalence of (5.4) with (5.2), see [2]. 


\section{REFERENCES}

1. E. Michael, Topologies on spaces of subsets, Trans. Amer. Math. Soc. 71 (1951), 152-182.

2. E. Michael and K. Nagami, Compact-covering images of metric spaces, Proc. Amer. Math. Soc. 37 (1973), 260-266.

3. V. Ponomarev, Axioms of countability and continuous mappings, Bull. Acad. Polon. Sci. Sér. Sci. Math. Astronom. Phys. 8 (1968), 127-134.

4. R. E. Smithson, First countable hyperspaces, Proc. Amer. Math. Soc. 56 (1976), 325-328.

5. H. H. Wiche, On the Hausdorff open continuous images of Hausdorff paracompact p-spaces, Proc. Amer. Math. Soc. 22 (1969), 136-140.

Department of Mathematics, Sasebo Technical College, Oxishin-Cho 1-1, Sasebo, NAGASAKI 857-11, JAPAN 